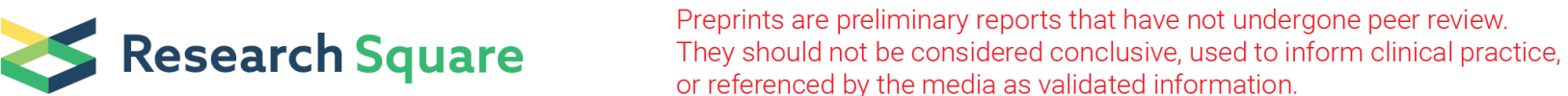

\section{Short and mid-term results of thoracoscopic surgery for esophageal atresia in low birth weight infants:A single-center clinical experience}

\section{Lei Yan}

Fujian Province Maternal and Child Health Hospital: Fujian Provincial Maternity and Children's Hospital https://orcid.org/0000-0001-7947-5989

MingKun Liu

Fujian Province Maternal and Child Health Hospital: Fujian Provincial Maternity and Children's Hospital

Bing Zhang

Fujian Province Maternal and Child Health Hospital: Fujian Provincial Maternity and Children's Hospital Yu Lin

Fujian Province Maternal and Child Health Hospital: Fujian Provincial Maternity and Children's Hospital

\section{Dianming Wu}

Fujian Province Maternal and Child Health Hospital: Fujian Provincial Maternity and Children's Hospital

YiFan Fang ( $\nabla$ Fyf0599@163.com )

Fujian Province Maternal and Child Health Hospital: Fujian Provincial Maternity and Children's Hospital https://orcid.org/0000-0003-3208-0482

\section{Research article}

Keywords: Esophageal atresia,Infant,Low birth weight,Thoracoscopy,Thoracotomy

Posted Date: March 26th, 2021

DOI: https://doi.org/10.21203/rs.3.rs-340827/v1

License: (a) (1) This work is licensed under a Creative Commons Attribution 4.0 International License. Read Full License 


\section{Abstract}

Purpose: To evaluate the short-term safety and long-term efficacy of thoracoscopic surgery for esophageal atresia(EA) in low birth weight infants.

Methods: From January 2011 to December 2019,a retrospective analysis on clinical data of 48 cases of low birth weight infants of EA. The clinical data were divided according to surgical methods: Thoracoscopy group A and thoracotomy group B. Variables of intra-operation, postoperative complications and mid-term postoperative complications were compared between the two groups.

Results: 3 were discharged due to treatment abandoning. there were 17 cases in thoracoscopy group $A$ and 28 cases in thoracotomy group B. The operation time of group $A(172.41 \pm 20.00$ min)was longer than $B(149.82 \pm 13.91 \mathrm{~min})$,the difference was statistically significant $(P<0.05)$.the intraoperative blood loss of group $A(7.41 \pm 2.83 \mathrm{ml})$ was less than $B(18.61 \pm 3.60 \mathrm{ml})$, the difference was statistically significant $(P<0.05)$. postoperative mechanical ventilation time, thoracic drainage time, hospital stay were not significantly different between the two groups. the incidence of anastomotic stenosis in group $\mathrm{B}(58.82 \%)$ was higher than in $A(28.57 \%)$, the difference was statistically significant $(P=0.045)$. There was no significant difference in the incidence of esophageal anastomotic fistula and recurrent tracheoesophageal fistula. After 3 years of follow-up, the incidence of thoracic deformity in group $B(25 \%)$ was higher than in $A(0 \%)$. The difference was significant $(P=0.034)$. However, no significant difference was observed among the gastroesophageal reflux, symptomatic stenosis, tracheomalacia.

Conclusion: Compared with thoracotomy, thoracoscopy in low weight infants with EA has the advantages of smaller incision, fewer intraoperative bleeding, and less incidence of thoracic deformities.

Thoracoscopy might be a feasible surgical option for low weight infants when performed by a surgeon who has rich experience. The major mid-term complications after surgery are esophageal stenosis, gastroesophageal reflux and tracheomalacia.

\section{Background}

Congenital esophageal atresia is a serious digestive tract malformation that occurs in 1 in $3000-4000$ newborns[1].The incidence in China is slightly lower than that in foreign countries. However,if not treated in time, it will seriously endanger the patient because of the large population. Prenatal color Doppler ultrasound of esophageal atresia often shows excessive amniotic fluid. The initial symptoms are foaming at the mouth,coughing,cyanosis,and trouble breathing. If not treated in time,most infants die in 3-5 days.At present,many single-center studies at home and abroad have reported that thoracoscopic surgery is a safe and feasible treatment for esophageal atresia,but most infants undergoing surgery have a preoperative weight greater than 2500 grams, who are resistant to thoracoscopic surgery and anesthesia.For infants with low birth weight,the safety and feasibility of thoracoscopic treatment and the long-term clinical prognosis remain under debate.Considering these circumstances,the purpose of this 
study is to analyze the surgical results of low birth weight infants in a single center,and to compare the efficacy and complications of thoracoscopy and thoracotomy.

\section{Methods}

\subsection{General data}

Select the clinical data of children with esophageal atresia hospitalized for 8 years from January 2011 to December 2019 in pediatric Surgery of fujian maternity and child health,and retrospectively analyze 48 cases of which received surgical treatment and were born Clinical data of children weighing less than $2500 \mathrm{~g} .3$ cases were excluded,who gave up treatment and were discharged automatically due to chromosomal abnormalities and multiple organ abnormalities found in preoperative examinations. With the maturation of thoracoscopic techniques and anesthesia for low birth weight infants,a number of thoracoscopic cases have gradually increased.According to the presurgical evaluation and the partents' willingness,they were divided into thoracoscopic surgery group and thoracotomy group,including 17 cases in thoracoscopy group and 28 in thoracotomy group.In the thoracoscopic group,there were 10 males and 7 females, with a birth weight of $2112.94 \pm 0.80 \mathrm{~g}$.According to the Gross method, 1 case was classified as type I, 1 case was type II,4 cases were type IIIA,and 9 cases were type IIIB.There were 5 cases of congenital heart disease, 3 cases of genitourinary system diseases, 3 cases of skeletal limb deformities, and 2 cases of anorectal gastrointestinal malformations. There were 15 males and 13 females in the thoracotomy group,with a birth weight of $2099.29 \pm 328.30 \mathrm{~g}$.According to Gross method,they were divided into 2 cases of type I, 1 case of type II, 6 cases of type IIIA, and 19 cases of type IIIB.There were 9 cases of congenital heart disease, 4 cases of genitourinary system diseases, 3 cases of skeletal limb deformities,and 5 cases of anorectal gastrointestinal malformations. There were no statistically significant differences between the two groups of children in gender,gestational age,age at surgery,birth weight,and whether they received mechanical ventilation before surgery (all $P$ value $>0.05$ )

(Table 1).Both groups of surgery were performed by physicians in the same treatment group.This study was approved by the Medical Ethics Committee of Fujian Provincial Maternity and Child Health Hospital.The guardians of the children all signed before the operation,confirmed their knowledge and signed the informed consent form for surgical treatment. 
Table 1

Baseline characteristics of two groups

\begin{tabular}{|c|c|c|c|}
\hline Group & $\begin{array}{l}\text { Thoracoscopy } \\
\text { Group(17) }\end{array}$ & $\begin{array}{l}\text { Thoracotomy } \\
\text { Group(28) }\end{array}$ & $\begin{array}{l}\mathrm{P} \\
\text { Value }\end{array}$ \\
\hline Gestational week(weeks) & $36.71 \pm 1.36$ & $36.93 \pm 1.59$ & 0.633 \\
\hline Birth weight(g) & $2112.94 \pm 0.80$ & $2099.29 \pm 328.30$ & 0.893 \\
\hline Operation age(day) & $3.24 \pm 1.35$ & $3.36 \pm 1.75$ & 0.807 \\
\hline $\begin{array}{l}\text { Preoperative ventilator } \\
\text { support(yes:no) }\end{array}$ & $4: 13$ & $10: 18$ & 0.392 \\
\hline Gender(Male:Female) & $10: 7$ & $15: 13$ & 0.731 \\
\hline IIIA:IIIB & $4: 9$ & $6: 19$ & 0.951 \\
\hline GROSS classification & 1 & 2 & \\
\hline I & 1 & 1 & \\
\hline II & 4 & 6 & \\
\hline IIIA & 9 & 19 & \\
\hline IIIB & 0 & 0 & \\
\hline IV & 0 & 0 & \\
\hline \multicolumn{4}{|l|}{ V } \\
\hline Number of malformations (Yes:No) & $6: 11$ & $13: 15$ & 0.463 \\
\hline Cardiac abnormalities & 5 & 9 & \\
\hline urogenital & 3 & 4 & \\
\hline Limb bones & 3 & 3 & \\
\hline Anal alimentary canal & 2 & 5 & \\
\hline
\end{tabular}

\subsection{Preoperative diagnosis}

All children with a gastric tube inserted through the nose were blocked from returning,and the esophagus was confirmed by esophageal angiography or esophageal CT three-dimensional reconstruction. The children were accompanied by various degrees of aspiration pneumonia before the operation.

\subsection{Surgical methods}

Surgical indications:Once the diagnosis of esophageal atresia is clear,surgical treatment is required,and esophageal end-to-end anastomosis can be performed by extrapleural or thoracic route. 


\subsubsection{One-stage angioplasty of type III esophageal atresia under thoracoscopy}

In the thoracoscopy group,the left side is lying,the body is tilted to the left about $45^{\circ}$ and the right upper limb is raised.Take the 4th intercostal space of the right subscapular corner line and place a $5 \mathrm{~mm}$ Trocar into the right thoracic cavity,establish artificial air pressure of $4-6 \mathrm{mmHg}(1 \mathrm{mmHg}=0.133 \mathrm{kPa})$, and place it on the 3rd and 3rd side of the axillary line under thoracoscopic monitoring.A $5 \mathrm{~mm}$ Trocar was placed in each of the 5 intercostal spaces as an operating hole.Find the odd vein after entering the chest.If the odd vein does not interfere with the operation,keep it,or free the odd vein for a section of about $1 \mathrm{~cm}$. Both ends are ligated with No. 1 silk thread and cut off.The chest wall is cut under the thoracoscopy.Find the distal esophagus on the right pleura of the posterior spine,and find the esophagotracheal fistula toward the trachea.Double-line ligation with No.1 silk thread and suture to cut off the esophagotracheal fistula.Ask the anesthesiologist to expand the lung and the right lung is well recruited.Find the proximal blind end of the esophageal atresia,free the blind end until it can be close to the distal end, remove the top and cut the blind end of the esophagus,use $5-0$ absorbable thread to intermittent valgus anastomosis to lock the distal and proximal ends,and insert the distal end after the anastomosis is completed.The gastric tube passes smoothly and then anastomoses the anterior wall of the two broken ends of the esophagus.

\subsubsection{Open chest type III esophageal atresia one-stage thoracotomy group}

take the left decubitus position, heighten the left chest,incision in the fourth intercostal space of the right chest wall,reach the front axillary line,and then reach the right subscapular corner line.After entering the chest,find the odd vein,separate the two ends of the odd vein with No.1 silk thread and cut it off,cut off the esophageal tracheal fistula $0.3 \mathrm{~cm}$ from the distal end of the ligation line,and sling at the distal and proximal ends of the esophagus by hanging and pulling at the bottom of the blind end of the esophagus.Make a transverse incision,pull out the preset stomach tube,and suture the far and near ends of the esophagus.

\subsection{Postoperative follow-up}

The children are followed up for the first 3 months after being discharged from the hospital,followed up once a month,during which at least one esophageal barium swallowing examination is performed. Thereafter,every 3 months to 1 year old,one esophageal barium swallowing examination is performed during the period.After 1 year of age,follow-up visits 1 to 2 times a year,and esophageal barium swallow angiography once a year.During the follow-up period,the esophageal barium swallow angiography was used to understand the status of the esophageal anastomosis,swallowing coordination,reflux,understand the morphological changes of the main trachea and the breathing process, and determine whether there is anastomotic stenosis and leakage,and whether there is gastroesophageal reflux,if necessary Gastroscopy,24-hour esophageal pH measurement,tracheal CT scan.Thoracic deformity is defined as skeletal deformity of the chest wall,such as depression of the chest wall,high scapula,rib fusion and scoliosis,etc.Anastomotic stenosis is defined as the presence of clinical symptoms (dysphagia,breathing disorder caused by aspiration or foreign body obstruction)and the stenosis can be found by endoscopy or 
esophagography (stenosis index $\geqq 0.5, \mathrm{SI}=1$-stenosis segment diameter/distal normal Diameter of the esophagus).

\subsection{Statistical processing}

Using SPSS 21.0 statistical software for data analysis,measurement data is represented by mean \pm standard deviation and range,independent sample t test is used for comparison between groups,and count data is compared by $x^{2}$ Test, $P<0.05$ indicates that the difference is statistically significant.

\section{Results}

\subsection{Two groups of surgery results}

All operations were successfully completed,and thoracoscopic surgery patients do not need open thoracotomy.There was no significant difference in the preoperative basic data,postoperative mechanical ventilation time, postoperative thoracic drainage time,postoperative hospital stay,etc.between the two groups $(P>0.05)$. However,the operation time of the thoracoscopy group was $(172.41 \pm 20.00 \mathrm{~min})$, and the operation time of the thoracotomy group was $(149.82 \pm 13.91 \mathrm{~min})$. The operation time of the thoracoscopy group was longer than that of the thoracotomy group.The difference in operation time between the two groups was statistically significant $(P<0.05)$.intraoperative blood loss in the endoscopic group $(7.41 \pm 2.83 \mathrm{ml})$,intraoperative blood loss in the thoracotomy group $(18.61 \pm 3.60 \mathrm{ml})$,intraoperative blood loss in the endoscopic group than in the thoracotomy group,and intraoperative bleeding in the 2 groups The difference in the amount was statistically significant $(P<0.05)$. (Table 2$)$.

Table 2

Variables of intra-operation of two groups

\begin{tabular}{|llll|}
\hline Group & $\begin{array}{l}\text { Thoracoscopy } \\
\text { Group(17) }\end{array}$ & $\begin{array}{l}\text { Thoracotomy } \\
\text { Group(28) }\end{array}$ & $\begin{array}{c}\text { V } \\
\text { Value }\end{array}$ \\
\hline Operation time(min) & $172.41 \pm 20.00$ & $149.82 \pm 13.91$ & $<0.05$ \\
\hline Amount of bleeding $(\mathrm{ml})$ & $7.41 \pm 2.83$ & $18.61 \pm 3.60$ & $<0.05$ \\
\hline Retain time of thoracic duct(day) & $11.12 \pm 2.71$ & $12.07 \pm 2.58$ & 0.245 \\
\hline Postoperative hospital stay(day) & $22.59 \pm 2.92$ & $22.21 \pm 3.92$ & 0.736 \\
\hline $\begin{array}{l}\text { Postoperative mechanical ventilation } \\
\text { duration(day) }\end{array}$ & $3.17 \pm 1.42$ & $3.32 \pm 1.79$ & 0.778 \\
\hline
\end{tabular}

\subsection{Complications and follow-up in the two group}

Combined with chest X-ray and CT examination. Among the postoperative complications,the incidence of anastomotic stenosis in the thoracoscopic group was $58.82 \%$, and the incidence in the open group was $28.57 \%$. The incidence of anastomotic stenosis in the thoracoscopic group was significantly higher than 
that in the open group, and the difference was statistically significant $(P=0.045)$. There was no statistically significant difference in the incidence of esophageal anastomotic fistula and recurrent tracheoesophageal fistula.After 3 years of follow-up,the incidence of thoracic deformity in the thoracotomy group was $25 \%$,which was significantly higher than the incidence of thoracoscopy at $0 \%$.The difference was statistically significant $(P=0.034)$. However,in the gastroesophageal reflux, symptomatic stenosis, The differences in tracheomalacia were not statistically significant.(Table 3 and Table 4).

Table 3

Short-term postoperative complications of two groups

\begin{tabular}{|c|c|c|c|c|c|}
\hline \multirow[t]{2}{*}{ Group } & \multicolumn{2}{|c|}{$\begin{array}{l}\text { Thoracoscopy } \\
\text { Group(17) }\end{array}$} & \multicolumn{2}{|c|}{$\begin{array}{l}\text { Thoracotomy } \\
\text { Group(28) }\end{array}$} & \multirow[t]{2}{*}{$\begin{array}{l}P \\
\text { Value }\end{array}$} \\
\hline & Yes & No & Yes & No & \\
\hline Esophageal anastomotic leakage & 2 & 15 & 5 & 23 & 0.693 \\
\hline Esophageal anastomotic stenosis & 10 & 7 & 8 & 20 & 0.045 \\
\hline $\begin{array}{l}\text { Recurrent oesophageal tracheal } \\
\text { fistula }\end{array}$ & 1 & 16 & 2 & 26 & 1.000 \\
\hline
\end{tabular}

Table 4

Mid-term postoperative complications of two groups

\begin{tabular}{|c|c|c|c|c|c|}
\hline \multirow[t]{2}{*}{ Group } & \multicolumn{2}{|c|}{ Thoracoscopy Group(17) } & \multicolumn{2}{|c|}{ Thoracotomy Group(28) } & \multirow[t]{2}{*}{ P Value } \\
\hline & Yes & No & Yes & No & \\
\hline Gastroesophageal reflux & 2 & 15 & 5 & 23 & 0.693 \\
\hline Tracheomalacia & 4 & 13 & 8 & 20 & 0.982 \\
\hline Chest deformity & 0 & 17 & 7 & 21 & 0.034 \\
\hline Esophageal stenosis & 7 & 10 & 6 & 22 & 0.281 \\
\hline
\end{tabular}

\section{Discussion}

Thoracoscope-assisted treatment of congenital esophageal atresia has a history of 20 years,but it started late in China,and it is still considered by many pediatric surgeons to be a very challenging operation[2, 3].Although thoracoscopic surgery has an obvious learning cycle, it has potential advantages,including small surgical incisions and low incidence of thoracic deformities.A meta-analysis of 48 articles found that thoracoscopic surgery to repair esophageal atresia and esophagotracheal fistula is safe.Compared with thoracotomy,the mortality rate in the thoracoscopy group did not increase.Although the time of 
thoracoscopic surgery is longer,the incidence of anastomotic leakage,postoperative esophageal stenosis and gastroesophageal reflux are comparable to those of thoracotomy[4].

At present,many international and domestic experts have proposed contraindications for thoracoscopic surgery,including long-spaced esophageal atresia,low birth weight children,and complicated congenital heart disease and severe pneumonia[5,6]. However,in clinical practice, it has been found that for children with low birth weight,if they are not combined with severe congenital heart disease and severe pneumonia,thoracoscopic surgery can also achieve good therapeutic effects[7].Therefore,this article separately analyzes the feasibility of thoracoscopic treatment for children with low birth weight, and also evaluates the long-term clinical prognosis and quality of life after surgery.

Low birth weight newborns pointed out that newborns with a birth weight of less than $2500 \mathrm{~g}$,due to the immature anatomy and physiological development of low birth weight newborns,many preoperative complications,poor tolerance to surgical anesthesia,high risk of anesthesia, and low weight This means that the child may have small physical growth and insufficient space for thoracic operation.Therefore,most doctors are unwilling to adopt thoracoscopic treatment. However,with the innovation of intraoperative anesthesia concepts and the maturity of intraoperative endoscopic operations, low birth weight newborns have more opportunities to receive thoracoscopic surgery, and the survival rate is increasing. Therefore,low body weight is not the main factor limiting thoracoscopic treatment.

Hypercapnia is one of the pathological conditions that are likely to occur in neonatal thoracoscopic surgery,especially in neonates with severe pneumonia before surgery or premature infants with pulmonary dysplasia[8, 9].In 2017,James K and others pointed out that carbon dioxide insufflation during thoracoscopic surgery may cause hemodynamic changes,such as hypotension and tachycardia.In addition,the establishment of a thoracoscopic pneumothorax may result in decreased blood oxygen saturation[7, 10].Finally,the absorption of $\mathrm{CO} 2$ by the pleura can lead to hypercapnia,the most serious consequence of which is metabolic acidosis. However,the proposed ventilation strategy for permissive hypercapnia solved this problem for clinicians. When using thoracoscopic surgery, choose a $\mathrm{CO} 2$ artificial pneumothorax pressure of 4-6 mmHg,which has less impact on the vital signs of newborns, is not easy to cause lung collapse,and also ensures a certain amount of chest operation space. There was no significant difference in the use time of the ventilator between the thoracoscopy group and the thoracotomy group,which may be related to the use of ventilation strategies for permissive hypercapnia during the thoracoscopy group to reduce lung compression.At the same time,pay attention to pneumonia before operation, clarify whether there is tracheomalacia,pay attention to lung management during operation,and select the appropriate surgical position,which will help improve the anesthesia tolerance of children[11]. While ensuring that the surgical field of vision is not disturbed,the blood oxygen fluctuation is maintained at about $90 \%$.

Thoracic surgery is a classic surgical method for the treatment of congenital esophageal atresia.However,the traditional surgical incision is long, the retractor is used to pull the ribs excessively 
during the operation, and the chest wall tissue is damaged[12].In addition to the long surgical scar left after the operation,the chest wall The incidence of musculoskeletal deformities is high,such as high scapula,rib fusion, and scoliosis[13]. while thoracoscopic surgery only needs to make three $5 \mathrm{~mm}$ holes in the chest wall,with small incisions, and little damage to the chest wall,intercostal muscles and nerves. The operation time of the thoracoscopy group was $172.41 \pm 20.00 \mathrm{~min}$, and the operation time of the thoracoscopy group was $149.82 \pm 13.91 \mathrm{~min}$. The operation time of the thoracoscopy group was longer than that of the thoracotomy group.The difference in operation time between the two groups was statistically significant $(P<0.05)$.It is very proficient with thoracotomy,but thoracoscopic surgery is difficult,requires high anesthesia, and needs to interrupt the operation waiting when the intraoperative percutaneous blood oxygen saturation drops. The intraoperative blood loss in the laparoscopic group was $41 \pm 2.83 \mathrm{ml}$, and the intraoperative blood loss in the thoracotomy group was $18.61 \pm 3.60 \mathrm{ml}$. The difference in intraoperative blood loss between the two groups was statistically significant $(P<0.05)$, which also confirmed the chest cavity The advantages of endoscopic surgery are less damage. The 3-year follow-up showed that the incidence of thoracic deformity in the thoracotomy group was $25 \%$,which was significantly higher than the incidence of $0 \%$ in the thoracoscopy group,and the difference was statistically significant $(P=0.034)$. The thoracoscopy has the function of magnifying the surgical field of vision,so that the surgeon's field of vision is clearer, the esophagus is freed more thoroughly, and the esophagus is sutured more finely[14].However,the newborn has a small chest cavity,small operating space,and large interference with the operation of breathing[15].Therefore,the surgeon is required to have skilled endoscopic operation experience and rich knowledge of pathological anatomy. The thoracoscopic technique can effectively preserve the odd veins.Our hospital has reported that esophageal atresia with thoracoscopic preservation of the odd veins can not only reduce the edema of the tissues around the esophagus,but also reduce the occurrence of esophageal anastomotic leakage and accelerate the postoperative recovery of children[16].We found that there was no significant difference in the incidence of esophageal anastomotic leakage between the thoracoscopy group and the thoracotomy group in the early postoperative period.It may be difficult to perform thoracoscopic surgery with children with lowweight esophageal atresia.In most cases,it is necessary to ligate the odd vein to facilitate The anastomosis is related to the esophagus,so the advantages of preserving the odd veins cannot be demonstrated.

Postoperative complications of esophageal atresia have been plagued by pediatric surgeons,affecting the quality of life of children.Its common complications include esophageal anastomotic leakage,anastomotic stenosis,gastroesophageal reflux,recurrence of esophageal anastomotic leakage,respiratory diseases and thoracic deformities[17].The main influencing factors for the occurrence of esophageal stenosis after esophageal atresia are the distance of the esophageal blind end,anastomotic leakage and postoperative gastroesophageal reflux[18]There is a difference in the incidence of early postoperative esophageal anastomotic stenosis between the two groups of thoracoscopy and open surgery. The incidence of thoracoscopy group is $58.82 \%$, which is higher than $28.57 \%$ of the thoracotomy group.Insufficient clipping of the proximal end of the anastomosis is related.At the same time,because of the obvious expansion of the proximal esophagus before the 
anastomosis,there is a certain degree of anastomotic stenosis in the early esophagus after esophageal atresia.Some studies believe that the stenosis(or asymptomatic stenosis)caused by early postoperative changes does not indicate the long-term stenosis formed without preventive dilation[19, 20].And most stenosis can be relieved by dilatation of the esophagus, so the two groups are comparable in the incidence of esophageal stenosis at 3 years after surgery.

Of course,our research also has certain limitations.Most of the collected cases of children weigh around $2000 \mathrm{~g}$, and there is a lack of research on children with very low birth weight undergoing esophageal atresia surgery.Andreas et al.proposed that the primary repair of type III esophageal atresia and tracheoesophageal fistula in very low and ultra-low birth weight newborns was not associated with the increase in intraoperative and postoperative complications compared with high birth weight newborns[21].Therefore, it is not necessary to give priority to staging surgery in these newborns,and should be selected according to the stability and degree of deformity of the child.According to our treatment experience, if the child does not have serious heart malformations and lung infections,even if the birth weight is extremely low,enteral nutrition through jejunostomy can be considered to be suitable for thoracoscopic treatment.

In summary, for low birth weight newborns, as long as there is no serious heart deformity and lung infection before surgery, they can tolerate intraoperative anesthesia, and through thoracoscopic surgery, they can achieve therapeutic effects equivalent to thoracotomy. The long-term curative effect is also comparable. At the same time, thoracoscopic treatment of esophageal atresia has the advantages of small surgical incision, less intraoperative bleeding and less incidence of thoracic deformities. It is safe and feasible, but it requires skilled thoracoscopic operation experience and anesthesiologist during operation. Coordination of airway management.

\section{Conclusions}

For low birth weight newborns,as long as there is no serious heart deformity and lung infection before surgery,they can tolerate intraoperative anesthesia,and through thoracoscopic surgery,they can achieve therapeutic effects equivalent to thoracotomy. The long-term curative effect is also comparable.At the same time,thoracoscopic treatment of esophageal atresia has the advantages of small surgical incision,less intraoperative bleeding and less incidence of thoracic deformities.It is safe and feasible, but it requires skilled thoracoscopic operation experience and anesthesiologist to coordination of airway management during operation.

\section{List Of Abbreviations}

EA esophageal atresia

\section{Declarations}


Acknowledgements

We highly acknowledge the contribution by the participating doctors:

Jianqin Zhang,Qiang Wu,Liu Chen,Yunjin Wang,Xu Cui,Wenhua Huang,Chaoming Zhou,Jianxi Bai

\section{Authors' contributions}

Lei Yan,Dianming Wu and Yifan Fang designed the study,collected the clinical data,performed the statistical analysis,participated in the

Operation,and drafted the manuscript.Mingkun Liu,Bing Zhang,Yu Lin participated in the operation and revised the article.All authors read and approved the final manuscript.

\section{Funding}

No funding.

\section{Availability of data and materials}

The datasets used and analysed during the current study are available from

the corresponding author on reasonable request.

\section{Ethics approval and consent to participate}

This study was approved by the ethics committee of our university and

strictly adhered to the tenets of the Declaration of Helsinki.In addition,all

patients' guardians signed an informed consent form before study.

\section{Consent for publication}

All the authors agree to the publication of this manuscript.

\section{Competing interests}

The authors declare that they have no competing interests.

\section{References}

1.Alter A, Almeida F, Mehta AC. Esophageal Atresia with Tracheoesophageal Fistula. American Journal of Respiratory \& Critical Care Medicine. 2016 : e7.

2.Zhang J, Wu Q, Chen L, Wang Y, Zhou C. Clinical analysis of surgery for type III esophageal atresia via thoracoscopy: a study of a Chinese single-center experience. J Cardiothorac Surg. 2020. 15(1). 
3.Van Lennep M, Singendonk MMJ, Dall'Oglio L, et al. Oesophageal atresia. Nat Rev Dis Primers. 2019. $5(1)$.

4.Way C, Wayne C, Grandpierre V, Harrison BJ, Nasr A. Thoracoscopy vs. thoracotomy for the repair of esophageal atresia and tracheoesophageal fistula: a systematic review and meta-analysis. Pediatr Surg Int. 2019. 35(3).

5.Holcomb, George W. Thoracoscopic surgery for esophageal atresia. Pediatr Surg Int. 2017. 33(4): 475481.

6.Shirota C, Tanaka Y, Tainaka T, Sumida W, Uchida H. Therapeutic strategy for thoracoscopic repair of esophageal atresia and its outcome. Pediatr Surg Int. 2019. 35(10): 1071-1076.

7.Wall JK, Sinclair TJ, Kethman W, et al. Advanced minimal access surgery in infants weighing less than 3kg: A single center experience. J Pediatr Surg. 2017 : S0022346817302713.

8.Pierro, Agostino. Hypercapnia and acidosis during the thoracoscopic repair of oesophageal atresia and congenital diaphragmatic hernia. J Pediatr Surg. 2015. 50(2): 247-249.

9.Augusto, Zani, Ruben, et al. Intraoperative acidosis and hypercapnia during thoracoscopic repair of congenital diaphragmatic hernia and esophageal atresia/tracheoesophageal fistula. Paediatr Anaesth. 2017 .

10.Koga $\mathrm{H}$, Yamoto $\mathrm{M}$, Okazaki T, et al. Factors affecting postoperative respiratory tract function in type-C esophageal atresia. Thoracoscopic versus open repair. Pediatr Surg Int. 2014. 30(12): 1273-1277.

11.van Hoorn CE, Costerus SA, Lau J, et al. Perioperative management of esophageal atresia/tracheoesophageal fistula: An analysis of data of 101 consecutive patients. Paediatr Anaesth. 2019.

12.Burford JM, Dassinger MS, Copeland DR, Keller JE, Smith SD. Repair of esophageal atresia with tracheoesophageal fistula via thoracotomy: a contemporary series. Am J Surg. 2011. 202(2): 203-6.

13.Bastard F, Bonnard A, Rousseau V, et al. Thoracic skeletal anomalies following surgical treatment of esophageal atresia. Lessons from a national cohort. J Pediatr Surg. 2017 : S0022346817304281.

14.Sanghoon, Lee, Suk-Koo, Lee, Jeong-Meen, Seo. Thoracoscopic repair of esophageal atresia with tracheoesophageal fistula: Overcoming the learning curve. J Pediatr Surg. 2014 .

15.Rozeik A, Elbarbary MM, Saleh AM, Khodary AR, Al-Ekrashy MA. Thoracoscopic versus conventional open repair of tracheoesophageal fistula in neonates: A Short term comparative study. J Pediatr Surg. 2019 .

16.Cui X, He Y, Chen L, et al. Clinical Analysis of Azygos Vein Preservation Under Thoracoscope in the Operation of Type III Esophageal Atresia. J Laparoendosc Adv Surg Tech A. 2020. 30(4): 448-452. 
17.Shah R, Varjavandi V, Krishnan U. Predictive factors for complications in children with esophageal atresia and tracheoesophageal fistula. Diseases of the Esophagus Official Journal of the International Society for Diseases of the Esophagus. 2015. (3): 216-223.

18.Nice T, Tuanama Diaz B, Shroyer M, et al. Risk Factors for Stricture Formation After Esophageal Atresia Repair. Journal of Laparoendoscopic \& Advanced Surgical Techniques Part A. 2016 : lap.2015.0120.

19.Yang YF, Dong R, Zheng C, et al. Outcomes of thoracoscopy versus thoracotomy for esophageal atresia with tracheoesophageal fistula repair: A PRISMA-compliant systematic review and meta-analysis. Medicine (Abingdon). 2016. 95(30): e4428.

20.Dai DL, Zhang CX, Zou YG, Yang QH, Wen FQ. Predictors of outcomes of endoscopic balloon dilatation in strictures after esophageal atresia repair: A retrospective study. World J Gastroenterol. 2020. 26(10): 1080-1087.

21.Schmidt A, Obermayr F, Lieber J, Gille C, Fideler F, Fuchs J. Outcome of primary repair in extremely and very low-birth-weight infants with esophageal atresia/distal tracheoesophageal fistula. J Pediatr Surg. 2017 : S0022346817303123. 